\title{
Estrategias DE APRENDIZAJE DESARROLLADOR EN LA FORMACIÓN MATEMÁTICA UNIVERSITARIA
}

\author{
DEVELOP OF LEARNING STRATEGIES IN UNIVERSITARY MATHEMATICS FORMATION
}

Dr. C. Ernesto V. Fernández Rivero Universidad Israel efernandez@uisrael.edu.ec

Mg. Yoandry Rivero Padrón Univerisdad Israel

Mg. Manuel S. Cañizares Jarrín Universidad Israel

Mg. Eduardo R. Pastás Gutiérrez Universidad Israel

Fecha de recepción: 14/05/2016 Fecha de Aceptación: 19/06/2016

\section{Resumen}

La preocupación por la poca durabilidad de contenidos matemáticos adquiridos por los estudiantes en etapas anteriores motivó a docentes de la Universidad Tecnológica Israel a buscar metodologías apropiadas que fomenten un aprendizaje significativo. El análisis de estudios recientes sobre esta temática condujo a metodologías que tienen en su centro el desarrollo de estrategias de aprendizaje significativo y desarrollador y su utilización en cursos de Matemática universitaria. 
Con sustento en una concepción de aprendizaje desarrollador (significativo y creativo), en el trabajo se describen herramientas para la estructuración de este proceso docente educativo.

Palabras clave: Aprendizaje, estrategias de aprendizaje, Aprendizaje desarrollador, Didáctica de Matemática, Matemática universitaria

\section{Abstract}

Concern over low durability of mathematical content acquired by students in previous stages motivated teachers of the technological University of Israel to seek appropriate methodologies that promote meaningful learning. Analysis of recent studies on this subject led to methodologies that have at its center the development of strategies of learning significant and developer and its use in courses of mathematical University.

With sustenance in a conception of developer (significant and creative), work-based learning tools for the structuring of the educational teaching process are described.

Keywords: learning, strategies for learning, developer learning, didactics of mathematics and University mathematics 


\section{Introducción}

En los cursos de Matemática en la Universidad Tecnológica Israel se observa en los estudiantes, poca durabilidad en el aprendizaje de contenidos matemáticos adquiridos, que, no pocas veces, es argumento de los bajos resultados docentes.

Con el fin de diagnosticar el estado real de esta problemática en la universidad se aplicó un cuestionario a estudiantes, que se complementaron con entrevistas a catedráticos del área Matemática, lo que evidenció:

- Los estudiantes esperan que el profesor oriente los pasos para resolver la tarea y ejecutan estos sin elaborar, previamente, un plan de solución;

- Algunos estudiantes reflexionan acerca de los conocimientos que poseen y que les permite resolver la tarea propuesta, pero no es frecuente hacerlo sobre estrategias cognitivas y metacognitivas que le facilitan llegar a encontrar una vía de solución;

- Ante la propuesta de solución de un compañero, el resto de los estudiantes la asumen como propia y dejan de buscar alternativas propias de solución;

- No acostumbran a buscar alternativas y reorganizar los pasos seguidos, de forma que puedan dar solución a la tarea;

- Es poco frecuente que los estudiantes expresen las respuestas a partir de determinar lo esencial y necesario;

- Los docentes no prestan especial atención al desarrollo de estrategias de aprendizajes efectivas.

Como el aprendizaje es el resultado de la interrelación de tres elementos clave: la intención (motivación) de quien aprende, el proceso que utiliza (estrategia) y los logros que obtiene (rendimiento), surge en un grupo de profesores el interés principal por estudiar estrategias de aprendizaje para mejorar la formación matemática de los estudiantes de la Universidad Tecnológica Israel. 
El informe que se presenta es un primer acercamiento a la problemática planteada y que puede servir de base a investigaciones que conduzcan a herramientas útiles a docentes y estudiantes para alcanzar aprendizajes significativos y desarrolladores, con metodologías renovadas para la conducción de este proceso.

\section{Hacia un aprendizaje significativo y desarrollador}

El resultado de investigaciones realizadas en los últimos 15 años por ha producido una profunda reconceptualización del vínculo entre los procesos de enseñanza, aprendizaje y desarrollo, enfatizándose en el carácter socializador, formativo y desarrollador del proceso de enseñanza-aprendizaje. Esta perspectiva integradora, desarrolladora, se corresponde con el significado que se ha dado nueva cultura del aprendizaje, que no solo exige nuevos tipos de aprendizajes, sino también nuevas formas de aprender dado que "aprender puede significar o requerir cosas distintas según las demandas culturales que lo motiven y el enfoque teórico que adoptemos.

Los procesos del aprendizaje desarrollador han sido concebidos como el resultado de la interacción dialéctica entre tres dimensiones básicas: la activación-regulación, la significatividad de los procesos y la motivación para aprender.

En investigaciones realizadas en el campo de la Matemática universitaria se evidencia la efectividad de las tres dimensiones básicas para el trabajo con estrategias metacognitivas y estrategias para la resolución de problemas en contenidos del Análisis Matemático y que recomienda su inclusión como contenidos de enseñanza, así como la estructuración de una colección básica de ejercicios utilizada para la fijación de los conocimientos y el desarrollo de las habilidades que ha logrado efecto positivo en relación con los saberes al pasar el tiempo.

Hay que tener en cuenta que el estudiante, al intentar resolver las tareas matemáticas que se le plantean, debe atender a:

- El dominio del conocimiento, que son aquellos contenidos estudiados en una disciplina matemática determinada y que pueden ser utilizados para resolver la situación planteada en la tarea; tales como definiciones, propiedades o leyes, procedimientos y concepción sobre las reglas para trabajar con el contenido matemático.

- Estrategias cognoscitivas, que incluyen métodos heurísticos; por ejemplo, descomponer el problema en casos simples, trabajo retrospectivo, invertir el problema, representar en diagramas o gráficas, el tanteo matemático, el uso de tablas y listas ordenadas, la búsqueda de patrones o relaciones y la reconstrucción del problema.

- Estrategias metacognitivas, que se relacionan con el monitoreo y el control. Están las decisiones globales con respecto a la selección e implementación de recursos y estrategias; es decir, acciones tales como planear, evaluar y decidir. 
- El sistema de creencias, que se compone de la visión que se tenga de las matemáticas y de sí mismo. Las creencias determinan la manera como se aproxima una persona a la tarea, las técnicas que usa o evita, el tiempo y el esfuerzo que le dedica, entre otras.

En interés de este trabajo, se centra la atención en las estrategias de aprendizaje, que constituyen procedimientos internos, no observables, de carácter generalmente cognitivo, que ponen en juego los sujetos cuando aprenden y que tienen como fin lograr un plan, un objetivo o una meta. Se distinguen de las estrategias de enseñanza, ya que las estrategias de aprendizajes son ejecutadas intencionalmente por un aprendiz (estudiante) siempre que se le demande aprender, recordar o resolver problemas sobre un contenido de aprendizaje.

En la literatura especializada se distinguen diferentes tipos de estrategias de aprendizaje: cognitivas, metacognitivas y de apoyo. Las estrategias cognitivas son procesos por medio de los cuales se obtiene conocimiento. Las estrategias metacognitivas son conocimientos sobre los procesos de cognición u auto administración del aprendizaje por medio de planeamiento, monitoreo y evaluación. Mientras, las estrategias de apoyo permiten al estudiante exponerse a sí mismo la asignatura que estudia y practicarla, explicarse y explicar a los demás, intercambiar ideas.

El aprendizaje es un proceso complejo, que ha sido objeto de estudio de diferentes concepciones (conductistas, cognitivistas, constructivistas y humanistas), cada una de las cuales ha realizado sus aportes a una mejor comprensión de dicho proceso, con diferencias esenciales y limitaciones.

La concepción desarrolladora e integradora del aprendizaje que se asume para el estudio de las estrategias se sustenta en posiciones de la Escuela Histórico-Cultural pues, es la más hospitalaria de las que existen hasta el momento en materia de psicología, porque su naturaleza dialéctica le permiten hospedar los mejores aportes de otros enfoques, con el ánimo de asimilarlos y crear una estructura teórica general más completa y profunda.

Cualquier tipo de aprendizaje produce cambios en determinados procesos y estructuras psicológicas internas y/o conductuales, que pueden ser observables o no externamente, a través de nuevas adquisiciones y logros, a través de períodos más o menos largos de la vida. Pero, solo en el caso de que esas nuevas adquisiciones encierren el potencial para favorecer nuevas transformaciones y por lo tanto para propiciar el tránsito del sujeto hacia niveles superiores de desarrollo, afirmamos que están ocurriendo aprendizajes que verdaderamente impactan el desarrollo integral y el crecimiento personal del individuo: aprendizajes desarrolladores que generarían, a partir de las propias contradicciones en que se fundamenta su existencia, la necesidad inagotable de aprender y de crecer, así como los recursos psicológicos personales cognitivos, afectivos, motivacionales-volitivos necesarios para lograrlo.

En consecuencia, se asume el aprendizaje humano, en un sentido amplio, como un proceso dialéctico de apropiación de los contenidos y las formas de conocer, hacer, convivir y ser construidos en la experiencia socio-histórica, en el cual se producen, como resultado de la actividad del individuo y de la interacción con otras personas, cambios relativamente duraderos y generalizables, que le permiten adaptarse a la realidad, transformarla y crecer como personalidad. 
Un aprendizaje desarrollador es aquel que garantiza en el individuo la apropiación activa y creadora de la cultura, propiciando el desarrollo de su auto perfeccionamiento constante, de su autonomía y autodeterminación, en íntima conexión con los necesarios procesos de socialización, compromiso y responsabilidad social.

Por tanto, para ser desarrollador, el aprendizaje tendría que cumplir con tres criterios básicos:

- Promover el desarrollo integral de la personalidad del educando, es decir, activar la apropiación de conocimientos, destrezas y capacidades intelectuales en estrecha armonía con la formación de motivaciones, sentimientos, cualidades, valores, convicciones e ideales. En otras palabras, garantizar la unidad de lo afectivo-valorativo en el desarrollo y crecimiento personal de los aprendices.

- Potenciar el tránsito progresivo de la dependencia a la independencia y a la autorregulación, así como el desarrollo en el sujeto de la capacidad de conocer, controlar y transformar creadoramente su propia persona y su medio.

- Desarrollar la capacidad para realizar aprendizajes a lo largo de la vida, a partir del dominio de las habilidades y estrategias para aprender a aprender, y de la necesidad de una auto-educación constante.

Los criterios básicos b y c en la concepción de una enseñanza en pos de un aprendizaje desarrollador presuponen asumir la enseñanza de estrategias no sólo cognitivas sino metacognitivas y en particular las referidas a la resolución de problemas y, para el desarrollo de la creatividad, convirtiéndolas de hecho en contenido de enseñanza. Otro elemento presente en este sentido lo constituye la elaboración, por parte del estudiante, de la orientación para la aplicación de procedimientos la cual es dada o elaborada, generalmente, por los docentes.

La educación matemática, tiene que ser una educación creativa, es decir, una educación que promueva un aprendizaje productivo y creador que fomente en los estudiantes una actividad científica y creativa ante la vida. Es imposible mejorar la educación matemática, desarrollar el pensamiento matemático de los alumnos en la resolución de problemas y otras actividades al margen de la creatividad.

Las técnicas de creatividad son métodos que permiten el entrenamiento creativo. Implican determinadas acciones que en general, son más importantes que la técnica en sí misma, y que sirven como estímulo.

Su análisis está estructurado según los siguientes ítems:

- Concepto

- Metodología para su utilización

- Consignas fundamentales y aspectos distintivos

- Aspectos relacionados con su utilidad, que guiarán la construcción de la clasificación a desarrollar. 
Las técnicas más usadas son: Uso de analogías, los seis sombreros para pensar, relaciones forzadas, matrices combinatorias, Ideart, entre otras.

Las clases de matemáticas abordan una parte teórica donde se presentan conceptos (mediante definiciones o descripciones), teoremas (que enuncian propiedades o leyes demostrando dentro de un sistema de conocimientos) y procedimientos para actuar (de tipo algorítmicos o heurísticos), pero una buena parte del tiempo se dedica a la práctica, es decir, a la fijación y aplicación del contenido matemático.

En los textos sobre Metodología de la enseñanza de la Matemática se precisa que un ejercicio (en sentido amplio) es una exigencia para actuar que se caracteriza por:

1. El objetivo de las acciones: transformar una situación inicial (lo dado) en una situación final (lo buscado).

2. El contenido de las acciones: objeto (elementos de materia matemática, su correspondencia con situaciones extramatemática y los elementos heurísticos a emplear) y tipos de acciones (identificar, realizar, comparar, ordenar, clasificar, reconocer, describir, aplicar, fundamentar, buscar, planificar, controlar).

3. Las condiciones para las acciones (exigencias que el ejercicio plantea al estudiante por su grado de dificultad).

El problema es un ejercicio que se caracteriza por tener una situación inicial conocida y una situación final desconocida, en que la vía de solución también es desconocida, pero que puede obtenerse con ayuda de procedimientos heurísticos.

Precisamente, la búsqueda de la vía de solución es la que resulta de mayor dificultad en los estudiantes. Para que un estudiante desarrolle estrategias de cognitivas y metacognitivas en la resolución de ejercicios y problemas, se debe conducir un proceso que contemple:

\section{Primer momento (tiene como objetivo final lograr una compresión del ejercicio o problema planteado)}

- La creación de una motivación (para una clase de ejercicios y que puede estar en el propio ejercicio y en las potencialidades de este para contribuir al desarrollo intelectual o la educación de los alumnos)-

- El planteamiento del problema (mediante las posibilidades o formas siguientes: encontrar el problema relacionado con la determinación de ciertas cantidades de magnitudes que surgen durante una discusión de clase; plantear una situación problemática que conlleva al planteamiento del ejercicio; entre otras).

- La comprensión del problema (con el empleo de impulsos como: lee el problema detenidamente; ¿De qué trata el problema?; formula el texto con tus propias palabras; observa figuras, tablas o esquemas 
dados en el problema o elabóralos, si fuera necesario; interpreta palabras claves o busca aclaración de términos desconocidos).

\section{Segundo momento (cuyo objetivo final es hallar la vía de solución).}

- La precisión del problema (a partir de la comprensión del problema se precisan los datos y lo buscado, comprende la formulación precisa del problema).

- El análisis del problema (orientado a una comprensión más profunda del problema en cuestión, a través de la interpretación y la traducción al lenguaje matemático de palabras claves se extraen relaciones y dependencias entre las magnitudes, posibles operaciones a realizar, posibilidad de relacionar magnitudes con ecuaciones o fórmulas conocidas; también es posible el empleo de medios heurísticos auxiliares y de procedimientos heurísticos).

- La búsqueda de la idea de solución (dirigido a la reflexión sobre los métodos que conducen al establecimiento de relaciones entre los datos y las incógnitas, que generalmente llevan al planteamiento de una ecuación o la aplicación de una fórmula; lo anterior permite la elaboración de un plan de solución en que están presentes las determinaciones de los medios matemáticos concretos y la aplicación de las estrategias de trabajo heurísticas).

\section{Tercer momento (orientado a resolver el ejercicio o problema planteado).}

- La ejecución del plan de solución (encaminado a la determinación del orden de realización de los cálculos, el análisis de realización del cálculo aproximado, análisis de las unidades de medidas, la utilización de magnitudes auxiliares. Debe tenerse presente los procedimientos heurísticos, en particular las reglas heurísticas).

\section{Cuarto momento (reflexión de lo aprendido en la resolución del ejercicio o problema).}

- El proceso concluye con reflexiones sobre la vía empleada para obtener la solución al problema, así como la comprobación de los resultados obtenidos.

La comprobación que se realiza con las relaciones que se establecen en el enunciado del ejercicio o mediante comparación de la posible solución con la estimación, el cálculo aproximado y la práctica, si resulta conveniente. Esto es lo que comúnmente realizan estudiantes y profesores y dan paso a nuevos ejercicios. Sin embargo, para el desarrollo de estrategias metacognitivas resulta de suma importancia la vía empleada para 
obtener la solución al problema. Se evalúa la vía de solución mediante consideraciones retrospectivas sobre procedimientos y métodos utilizados para el plan de solución, se reflexiona sobre otras vías de solución.

En los procesos de resolución de ejercicios y problemas en que está presente el docente es importante que éste ofrezca impulsos heurísticos en los pasos de búsqueda de una solución, así como en la reflexión final en la evaluación de la vía empleada que permite llegar a situación final deseada (la solución).

Como resultados de investigaciones realizadas este sentido se recomienda a los docentes, que al elegir los contenidos de un tema:

- Seleccionar la colección de ejercicios que por su concepción garantiza fijar los conceptos, teoremas y procedimientos, así como un desarrollo de las habilidades que se mantienen al pasar el tiempo, es aquella que posee, entre otros, ejercicios cuya situación final exige probar que se cumple, que no se cumple o determinar las condiciones en que se cumple o no para un objeto si pertenece a una clase, o en iguales condiciones de exigencia el cumplimiento de una propiedad o la ejecución de un procedimiento.

- Determinar las estrategias del pensamiento que deben ser enseñadas, que son aquellas que garantizan entre otros aspectos mayor control sobre los procesos que se realizan, más conciencia de lo que se sabe y de lo que aún no se sabe, que permiten valorar mejor lo que se hace, lo que no se hace y lo que hacen los otros, que permiten, en resumen, regular la actividad propia.

\section{Ejemplo}

\section{Ante una exigencia:}

- ¿Qué me piden?

- ¿Qué se de ello?

- ¿Qué necesito utilizar para responder?, ¿una definición?, ¿un teorema?, ¿un principio?, ¿una ley?, ¿una propiedad?...

- ¿Cómo se relaciona lo que me piden con lo que determiné utilizar? ¿Estoy haciéndolo bien? Debo chequearlo, no me da y no tengo errores, debo cambiar.

\section{Para encontrar una vía de solución debes:}

- Interpretar lo buscado en todas las formas posibles y relacionarlo con lo que sabes.

- Seleccionar de lo que sabes, lo que necesitas para inferir lo buscado (condición necesaria, condición suficiente, procedimiento). 
- Determinar de lo que te dan, qué falta para inferir lo buscado según la condición necesaria o suficiente o el procedimiento que seleccionaste.

Se debe, entonces, determinar el método para el proceso de enseñanza-aprendizaje significativo, desarrollador y creativo que mejor propicie:

- Desarrollo del pensamiento.

- Aprendizaje activo, cooperado y flexible.

- Formar o desarrollar valores (personales y sociales).

- Dominio de la Matemática (su estructura, invariantes, solidez de los conocimientos, habilidades desarrolladas, interdisciplinariedad).

- Investigar. Vincular, en lo posible, con la práctica.

- Desarrollar la capacidad comunicativa, reflexiva y autorreflexiva, a partir de:

- escuchar críticamente en función de diálogos reflexivos.

- enseñar a interpretar.

- enseñar a fundamentar y refutar.

- buscar soluciones eficientes.

- reflexionar y criticar colectivamente.

- comprender cómo se piensa, qué se sabe, qué no, aciertos y deficiencias, causas.

- evaluar y calificar colectivamente.

- Enseñar a aprender.

La evaluación como proceso para comprobar y valorar el cumplimiento de los objetivos propuestos y la dirección didáctica de la enseñanza y el aprendizaje en sus momentos de orientación y ejecución, deberá proponer actividades cuyo contenido exprese exigencias en correspondencia con los objetivos trazados e incorpore la autoevaluación por los estudiantes, así como las acciones de control y valoración del trabajo a través de:

- La disposición por participar.

- La entrega de tareas.

- El avance del estudiante en su aprendizaje.

- Las preguntas realizadas.

- Uso de diferentes vías de solución.

- Control e interpretación de resultados.

- Utilización de estrategias tanto cognitivas como metacognitivas.

- Tiempo utilizado al resolver un ejercicio o problema.

- El éxito en la resolución del ejercicio o problema. 
Lo expresado en este trabajo permite concluir que, los contenidos declarados en los sílabos de los cursos de Matemática son el punto de partida para el desarrollo de un aprendizaje significativo, desarrollador, creativo, pero se requiere, además, conducir el proceso de aprendizaje de manera adecuada, entonces, es necesario renovar y perfeccionar las metodologías (estrategias de aprendizaje) empleadas, a la realidad actual de la formación universitaria en la Universidad Tecnológica Israel. 


\section{Bibliografía}

Ballester, S. et al. (1992). Metodología de la enseñanza de la Matemática (Tomo I). Editorial Pueblo y Educación. Ciudad de la Habana.

Ballester, S. et al. (2000). Metodología de la enseñanza de la Matemática (Tomo II). Editorial Pueblo y Educación. Ciudad de la Habana.

Biggs, J. (1988). Approaches to learning an to essay writing. (R. S. (Ed.), Ed.) Learning strategies and learning styles.

Castellanos Simons, D. (2007). Teorías actuales del aprendizaje. Caracas, Venezuela: Imprenta Universitaria UBV.

Castellanos Simons, D., Castellanos Simons, B., Llivina Lavigne, M. J., \& colectivo, S. M. (2002). Aprender y enseñar en la escuela: una concepción desarrolladora. La Habana: Pueblo y Educación. Obtenido de http://esdocs.org/docs/index-16868.html

Cevallos Gamboa, W. A. (22 de Octubre de 2010). DSPACE ESPOL. Obtenido de DSPACE ESPOL: http://www.dspace.espol.edu.ec/ bitstream/123456789/13388/1/Resumen_Tesis_CICYT-ESPOL_Antonio_Cevallos\%5B1\%5DULTIMA.pdf

Colectivo de docentes Universidad de Matanzas. (2000). Técnicas Creativas. La Habana, Cuba: Editorial Universitaria.

Delgado Vilela, T. P. (2013). DSSPACE ESPOL. Obtenido de DSSPACE ESPOL: http://www.dspace.espol.edu.ec/handle/123456789/24826

Fariñas, G. (2001). Psicología Educativa. Selección de lecturas. La Habana., Cuba: Félix Varela.

Jiménez, H. M. (2010). Metodología para la instrumentación del enfoque desarrollador en el proceso de enseñanza-aprendizaje de la Matemática en la Educación Superior. ADMES. La Habana: Informe sobre resultado de investigación. Universidad de Ciencias Pedagógicas "E. J. Varona”.

Lopez, D. (1 de Octubre de 2009). buenosaires. Obtenido de buenosaires: http://www.buenosaires.gob.ar/areas/educacion/niveles/ especial/documentos.php?menu_id=32092

Matemática, M. R. (Ed.). (s.f.). Blog de Formación Inicial Docente. Obtenido de http://www2.minedu.gob.pe/digesutp/formacioninicial/

Monereo Font, C. (1984). Estrategias de aprendizaje y enseñanza. (Colección de aprendizaje ed.). (U. d. Girona, Ed.) Catalunya, España: Eds. Universitat.

PLATA ALARCÓN, W. R. (2009). DSPACE ESPOL. Obtenido de DSPACE ESPOL: https://www.dspace.espol.edu.ec/handle/123456789/8104

Pozo, J. I. (1985). Teorías cognitivas del aprendizaje. (10ma Edición. ed.). Madrid, España: Ediciones Morata. Obtenido de http://www. edmorata.es/libros/teroias-congnitiva-del-aprendizaje. 
Pozo, J. I. (1998). Aprendices de maestros. La nuev cultura del aprendizaje. Madrid: Alianza Editorial.

Pozo, J. I. (1998). Aprendices y maestros. La nueva cultura del aprendizaje. Madrid: Alianza Editorial.

Santos, L. M. (1992). El trabajo de Alan Schoenfeld: Una propuesta a considerar en el aprendizaje de las Matemáticas. Educación Matemática, V. 4(No. 2).

Schoenfeld, A. H. (1985). Ideas y tendencias en la resolución de problemas. La enseñanza de la Matemática a debat. Madrid, España: Ministerio de Educación y Ciencia.

Universitat. (14 de Noviembre de 2014). Universitat. Obtenido de Universitat: http://fci.uib.es/Servicios/libros/veracruz/nasso/Proceso-de-Ensenanza-Aprendizaje-en-Educacion.cid210315 
